\title{
DESIGN MANAGEMENT: METRICS AND VISUAL TOOLS
}

\author{
Hisham Abou-Ibrahim ${ }^{1}$ and Farook Hamzeh ${ }^{2}$
}

\begin{abstract}
The iterative and multidisciplinary nature of design complicates its management. Nonetheless, the lack of adequate tools that can be used to manage design dynamics negatively affects the design process as well as the quality of the final design deliverables. In this regard, this paper introduces new metrics to measure information flow in BIM projects, and elaborates on the Level of Development (LOD) concept to reflect the design maturity of model elements in the corresponding design context. Moreover, a metric is developed to reflect the design maturity of the entire BIM model or part of it. The study also employs visual tools to plan and control design tasks based on the developed metrics. Results highlight that quantifying and visualizing the design progress helps design managers better assess the design status, streamline information flow among parties, and control the generation of corresponding design information.
\end{abstract}

Keywords: Lean design, Visual Planning, BIM, LOD.

\section{INTRODUCTION}

Design is a multidisciplinary process that aims to fulfil owner's needs and values by overcoming several obstacles and constraints (Cross 1984). Problems with design management can be attributed to the iterative nature of design, the involvement of different parties with different interests and mentalities, poor communication and inadequate information exchange, segregated delivery systems, and the absence of adequate metrics that can quantify design information flow using real time data (Ballard 2000, Tribelsky and Sacks 2011, Ford \& Sterman, 2003).

Meanwhile, the industry is witnessing an increasing use of Building Information Modelling (BIM) as a platform for running the design process. BIM is a visual database that combines parametric design data into a centralized model. The proper use of BIM has been proven beneficial for the design process as well as the final design product (Eastman, et al. 2009; Al Hattab and Hamzeh 2016). Since BIM is object oriented, elements used in the model holds the corresponding design information either in their graphical appearance or in the corresponding attached data. Accordingly, managing design data on BIM defers from traditional 2D-CAD processes.

As research on design management is gaining momentum in different directions, visualizing and quantifying the planning and control of the design process is expected to streamline the flow of information among stakeholders, reduce waste and rework, promote coordination among involved stakeholders, and increase the quality of design for downstream and end customers.

PhD. Student, Civil and Environmental Engineering, American University of Beirut, Lebanon, +961 3 900695, haa131@mail.aub.edu.

2 Assistant Professor, Civil and Environmental Engineering, American University of Beirut, Lebanon, + 9611350000 ext 3616, fax: + 611 744462, Farook.Hamzeh@aub.edu.lb. 


\section{LITERATURE REVIEW}

\subsection{Waste in Design}

An interesting study by Mazlum and Pekericli (2016) underlines the wastes encountered during the design process, and classifies them according to Ohno's waste categories. Results show that waste related to rework is the most frequent category and has the highest cost impact. Koskela et al. (2002) refer to two main reasons that cause rework: (1) unclear definition and poor ordering of design tasks, and (2) failure to stick to the ideal order, in case planned, due to external enforcing factors. Consequently, design tasks usually suffer from a lack of timely information exchange. In this regard, Tribelsky and Sacks (2011) claim that focusing on the information flow and data sharing characteristics can reduce the generation of waste and increase the value for downstream and end customers.

To minimize rework, the Dependency Structure Matrix (DSM) tool is used to order design tasks according to the corresponding information dependency (Austin et al. 1999). Accordingly, better flow of information among design parties is expected. However, scheduling the design program resulting from the DSM requires not only the sequence of activities, but also the start/end dates, durations, and resources requirements of each activity. Accordingly, the lookahead planning technique is suggested to further detail design activities, identify constraints, allocate resources and release work packages. Nonetheless, the Percent Plan Complete (PPC) was used to manage projects during the design phase (Hamzeh et al. 2009, Hammond et al. 2000). However, the characteristics of the design process cannot be fully captured in these frameworks. For example, PPC only measures the quality of the scheduled plan not the quality of design itself. A $100 \%$ PPC does not necessarily reflect a $100 \%$ accepted design. Moreover, these techniques focus on the flow aspect of design and gives less attention to its transformation and value characteristics.

\subsection{Design Information Flow}

In a different direction, several studies target the definition of information flow during the design process. Note that the conceptualization of information flow during design governs to a big extent the way design is being planned and monitored. For instance, Krovi et al. (2003) compares information flow to fluid mechanics where characteristic such as velocity, viscosity, and volatility are proposed, but without being measured or demonstrated. Ballard (2000) approaches design workflow as the flow of products in manufacturing, where designers transform client requirements and ideas into product design documents. Tribelsky and Sacks (2010) further elaborate on Ballard's model and introduce metrics to quantify design workflows. These metrics help evaluate the characteristics of information flow such as work in process, cycle time, batching and other lean related concepts. However, these metrics do not reflect the quality of design itself nor its progress, they just reflect the characteristics of the ongoing process.

\subsection{Level of Development (LOD)}

To manage information flow in BIM, research and industry efforts created the notion of Level of Development (LOD). LOD is seen as a communication language among design participants to formalize the development of model elements and control their use by downstream users. LOD defines the minimum content requirements for an element and its authorized uses at five progressively detailed levels of completeness. Current 
classification systems range from LOD 100 to LOD 500, specifying the minimum graphical and non-graphical information an element should hold at each level, and its possible authorized uses (BIMForum 2015; The American Institute of Architects 2013).

In this context, an LOD-based framework is developed by Abou-Ibrahim and Hamzeh (2016) to expand the use of LOD into design management, not just model management. The framework creates three variables to describe LOD, and introduces a matrix to relate LOD to these variables. The framework allows stakeholders to prioritize between graphics and information while deciding on modeling requirements. Also, a new variable is introduced to describe the different design checks performed on the element during the process, as a different approach to defining an element's design reliability. However, some practitioners and academics suggest abiding by the available LOD guidelines to avoid inconsistencies, especially that these guidelines are used contractually.

In this regard, a new method to plan, schedule, and control design activities is needed. This paper suggests a framework to manage design using BIM, and the objectives of the study can be stated as follows:

1. Define design workflow on BIM platforms

2. Define metrics to quantify the defined information flow

3. Visualize the planning and control of the design process

To realize these objectives, the study introduces the Design Maturity Index (DMI) that describes the design maturity of the BIM model, a discipline, or even a certain category of elements. DMI is based on the LOD concept, and expands its use for design management purposes. Thus, while LOD reflects the modelling requirements of an individual model element, DMI targets the design maturity of the entire model or part of it. An equation is developed to calculate DMI, and visual tools are used to plan and control the design process.

\section{Methodology}

Design Science Research (DSR) is used as a research method in this study. DSR is sometimes called "improvement research" to emphasize the problem solving nature of the activity. DSR starts by recognizing and defining the problem. Then, suggestions for a problem solution are drawn from the existing knowledge of the problem area (Peirce 1931). Afterwards, implementing the suggestions is performed under the Development phase, to be afterwards evaluated according to the functional specification implicit or explicit in the suggestion. Development, Evaluation, and further Suggestions are often iteratively performed in the course of the research effort. Conclusion indicates termination of a specific research project (Vaishnavi and Kuechler 2007).

\begin{tabular}{|c|c|c|c|c|}
\hline $\begin{array}{l}\text { Awarness of the } \\
\text { problem: Lack of } \\
\text { ppropriate methods } \\
\text { hat can be used to } \\
\text { lan, schedule and } \\
\text { ontrol the design } \\
\text { rocess using BIM }\end{array}$ & $\begin{array}{l}\text { 2. Suggestion: } \\
\text { I I I that can quantify and } \\
\text { I I visualize design } \\
\text { information flow } \\
\text { I I Ising the LOD } \\
\text { I I concept and newly } \\
\text { I I } \\
\text { I I }\end{array}$ & $\begin{array}{l}\text { 3. Development: } \\
\text { | Conceptualize } \\
\text { | design workflow as a } \\
\text { | flow of model } \\
\text { elements inside BIM | } \\
\text { models, and develop | } \\
\text { the corresponding }\end{array}$ & 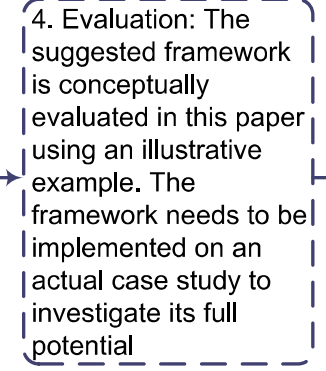 & $\begin{array}{l}\text { usion: the } \\
\text { d } \\
\text { rk helps } \\
\text { and } \\
\text { the }\end{array}$ \\
\hline
\end{tabular}

Figure 1: Research method using DSR 


\section{FRAMEWORK DEVELOPMENT}

\subsection{Conceptualization of Information Flow in BIM}

When designers employ BIM during design, they actually create parametric elements in the model. These elements are classified by categories, families, and types as illustrated in Figure 2. The following are the definitions of these classes as they appear in Autodesk:

- Category: it is a group of elements used to model building design, for example columns, beams, walls, etc (Autodesk n.d.).

- Family: it is a class of elements under a certain category. "A family groups elements with a common set of parameters (properties), identical use, and similar graphical representation. Different elements in a family may have different values for some or all properties, but the set of properties-their names and meaning-is the same" (Autodesk n.d.). For example, a lighting fixture family can have pendant lights coming in different sizes and material, but having similar shapes.

- Type: it is a specific case of a family, where specific values are given to size, material, or any property. Every family can have several types (Autodesk n.d.).

- Instance: it is the actual element placed in the model and have a specific location.

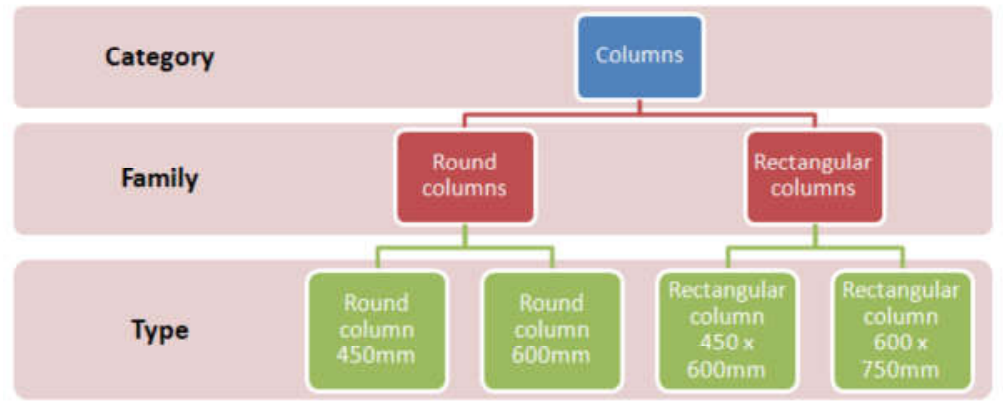

Figure 2: Hierarchy in BIM models (Autodesk, n.d.)

Design workflow in BIM projects is hereby approached as a flow of model elements. At every stage in design, some new elements are created, other elements are further developed, and some elements are deleted or changed. Thus, design dynamics among stakeholders is reflected by the generation and development of model elements throughout the process. In this regard, design information is being delivered through these elements either by their graphical appearance or their attached non-graphical data. The following is a set of notions used in this paper to describe information flow in BIM:

- Information Package: it can be the full BIM model, a discipline model, a category of elements or any part of the model used to deliver design intent.

- Element: it is the smallest model entity used to hold and deliver design information.

- Design Information: it is any graphical or non-graphical data attached to an element.

- LOD: it is the Level of Development of a model element based on its graphical appearance, attached information, and authorized uses.

- EDM: it is the Element Design Maturity based on the corresponding design context.

- DMI: it is the Design Maturity Index of the entire model or part of it. 


\subsection{Element Design Maturity (EDM)}

The LOD classification system is currently used to describe model elements according to their graphical appearance, attached information, and authorized uses. However, current LOD guidelines are not oriented to reflect design related conditions witnessed throughout the process. For instance, at a certain stage in design, an element or set of elements in the model may be waiting for owner's approval, coordination with other disciplines, or even acceptance from concerned authorities. Although these elements might have a high LOD level (say LOD 300), their design maturity cannot be directly deduced since it is related to a bigger design context involving several stakeholders.

To account for these important aspects witnessed during design, the Confidence Index (CI) metric introduced by Abou-Ibrahim and Hamzeh (2016) is used in this paper. CI represents the design reliability of each element used in the BIM model and it is based on Gray and Hughes (2001). The design checking process can be divided into two main categories: (1) reviews targeting client needs vs. building standards and (2) reviews targeting product's in-service requirements (Gray and Hughes 2001), as highlighted in Table 1. For example, when an element is checked against client's value, the element gains $\mathrm{C} 1$. If in addition, the element is checked against other disciplines, it gains C4. CI can take ten different values ( $\mathrm{C} 1$ to $\mathrm{C} 10)$ according to each review type. The mentioned types are suggested to generally describe the checking process happening at the design stage.

In this context, a metric called Element Design Maturity (EDM) is developed to reflect the design maturity of an element based on its LOD level and CI values. While LOD reflects the level of development of an element based on its modeling characteristics, EDM reflects the design maturity of the element based on the design context. EDM has five maturity levels as highlighted in Table 2.

Table 1: Different design checks performed under CI (Gray and Hughes 2001)

\begin{tabular}{|c|c|}
\hline Review Type & Reliability Check Type \\
\hline \multirow{5}{*}{$\begin{array}{l}\text { Reviews targeting client } \\
\text { needs vs. building } \\
\text { standards }\end{array}$} & C1: Client needs vs. standard or innovative technical specifications. \\
\hline & $\begin{array}{l}\text { C2: Compliance with building regulation, planning regulations, health and } \\
\text { safety law, national and international standards. }\end{array}$ \\
\hline & C3: Building Performance under expected conditions of use. \\
\hline & C4: Design validation and coordination among different trades. \\
\hline & C5: Building safety and environmental compatibility. \\
\hline \multirow{5}{*}{$\begin{array}{l}\text { Reviews targeting } \\
\text { product's in-service } \\
\text { requirements }\end{array}$} & C6: Constructability. \\
\hline & C7: Permissible assembly tolerances. \\
\hline & C8: Failure modes and effects, and fault analysis. \\
\hline & C9: Reliability, serviceability, and maintainability of building elements. \\
\hline & $\begin{array}{l}\text { C10: Labeling, warnings, identification, and traceability requirements of } \\
\text { building elements. }\end{array}$ \\
\hline
\end{tabular}

Table 2: Element Design Maturity Levels

\begin{tabular}{llll}
\hline EDM Level & $\begin{array}{l}\text { LOD } \\
\text { Requirements }\end{array}$ & CI Requirements & Maturity Level \\
\hline EDM-1 & LOD 100 & C1 & Conceptual \\
\hline EDM-2 & LOD 200 & C1, C2, C4 & Schematic \\
\hline EDM-3 & LOD 300 & C1, C2, C3, C4, C5 & Detailed \\
\hline EDM-4 & LOD 400 & C1, C2, C3, C4, C5, C6, C7, C8 & Shop Model \\
\hline EDM-5 & LOD 500 & C1, C2, C3, C4, C5, C6, C7, C8, C9, C10 & As-Built \\
\hline
\end{tabular}




\subsection{Design Maturity Index (DMI)}

Since EDM reflects the design maturity of an individual element, Design Maturity Index (DMI) is developed to reflect the design maturity of the entire model or part of it. DMI is the ratio of elements that reached their desired EDMs to overall modeled elements. For instance, if the planned maturity level of the architectural model is Schematic, designers need to model architectural elements according to LOD 200 requirements and check their model against client's needs (C1), planning and regulation rules (C2), and other involved disciplines at the moment (C4). Equation (1) is used to calculate the DMI of a set of model elements. DMI ranges between 0 and 100 where none or all modeled elements have converted to their planned EDMs.

Note that designers are not aware of the final number of elements to be modeled at the end of design; however, they know that all of these elements should abide by the corresponding LOD and CI requirements originally planned. This is why the percentage is used not the number of elements. Thus, DMI evaluates the ratio of elements that reached their EDM requirements based on the total number of elements modeled at the moment. Although both the nominator and denominator of the DMI equation would be continuously evolving especially at early design phases, DMI would still reflect the actual model design maturity at the corresponding moment with respect to the original EDM plan. Practically speaking, designers are not required to figure out the EDM of each element separately; however, they can benefit from the parametric structure of the model to automate this task. Rule Based checking algorithms can be developed for this purpose to fully automate the procedure.

$$
D M I=\frac{\sum \text { Elements that reached planned EDM }}{\text { Total number of elements in the defined set }}
$$

\subsection{Visual Planning and Control}

Using the definition of information flow provided in Section 4.1, a new method to plan, schedule, and control design development is proposed in this section. Since design encompasses a flow of elements, design managers can plan the development of these elements at each phase of the design process based on the desired maturity level. For instance, at the schematic phase of design, managers can agree to deliver architectural and structural elements at EDM-2, while designing MEP elements at EDM-1. Note that we can have elements at different EDMs at each phase of the project.

Since elements are distributed among categories as highlighted in Figure 2, practitioners can plan the progress of design using these categories as Information Packages. They can decide on the categories to be designed in each phase, the EDM requirements of corresponding elements, the sequence, the start/ end dates, the durations, and the responsible design teams. These categories are planned to reach a $100 \%$ DMI at the end of their corresponding durations, where all sub elements are required to reach their planned design maturity. Note that other Information Packages can be used to schedule the design process, for example disciplines, systems, or other parts of the model.

To visualize the planning of design, a method inspired by the Line of Balance technique is suggested. However, instead of locations, DMI percentages are used on the Y-axis as highlighted in Figure 3. This method is referred to as Design Flow Lines (DFL) in this paper. Each DFL reflects the start date of a certain design activity, its end date, the corresponding duration, and its pace. Each DFL starts by a DMI of zero and ends up with a DMI of 100 where all corresponding elements are planned to reach a certain design maturity. 


\section{FRAMEWORK EVALUATION}

An illustrative example is used to evaluate the suggested framework at its theoretical level. Note that a real case study is needed to further evaluate the practicality and usefulness of the framework. Figure 3 illustrates the use of DFLs to plan the design process using elements' Categories. Dashed Lines reflects the original plan, whereas solid lines reflect the actual DMI values. Architectural Walls and Floors are two Categories used to design spaces, overall building shape and function. These Categories are interdependent (form a loop in the DSM), and therefore they are coupled together in one DFL. They are planned to start at week 0 and reach their desired design maturity at week 4 . Rooms are another architectural Category used to annotate the designed spaces. Rooms design is planned to start one week before the Architectural Walls and Floors reach 100\% DMI. The design of Columns also has a one-week overlap with Architectural Walls and Floors, and it is expected to reach $100 \%$ DMI in two weeks. Floor Openings are designed by MEP engineers while deciding on the MEP concept. In this example, their start date is planned after the architectural and structural engineers finish their designs.

Once the plan is set (dashed lines), design managers can follow up with design progress by calculating the actual DMIs (solid lines) at the end of each week. Recall that DMI is the percent of elements that reached their desired EDMs to the total number of elements in the Category. For instance, Architectural Walls and Floors failed to reach their planned design maturity by week 4, and witnessed a one week of delay. This delay affects the completion date of Columns' design where structural engineers needed to wait for architects to finalize the building spaces before they can finalize the columns' grid. Nonetheless, MEP engineers waited for one week before they could start allocating Floor Openings; however, the corresponding duration was not affected. Note that Rooms were not modeled, and the visualization made it easy to detect this deviation from the plan.

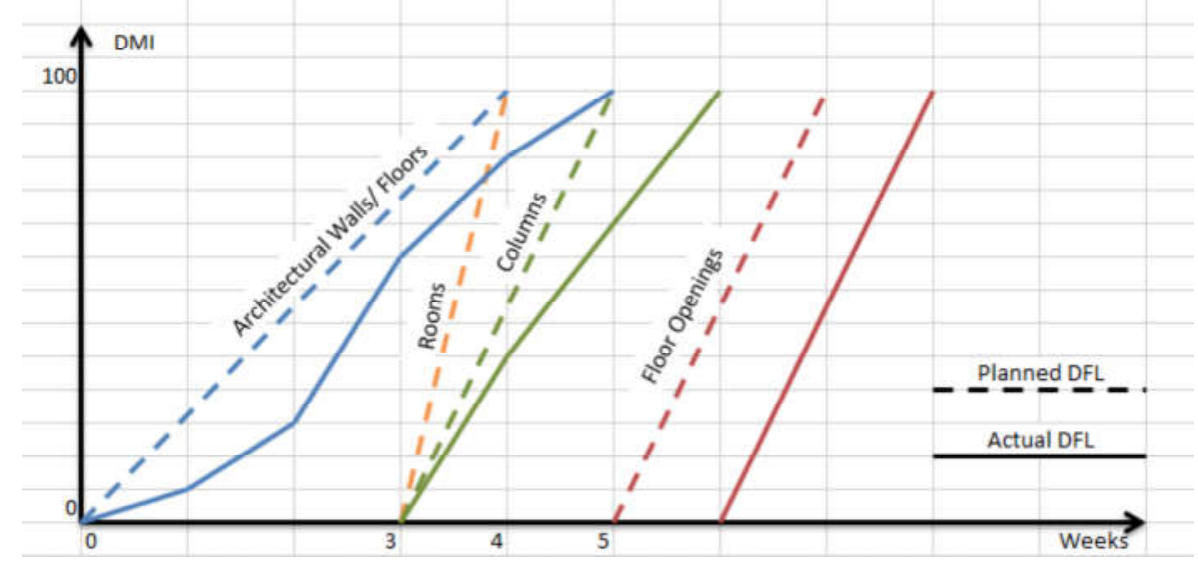

Figure 3: DFL plan for design development

\section{DISCUSSION AND CONCLUSIONS}

A new design management framework is developed in this study to enhance the planning and control of the design process. The framework builds upon the LOD concept, develops new design progress metrics, and uses Design Flow Lines (DFLs) to visualize the planning and control of design using BIM platforms. 
In this regard, the Element Design Maturity (EDM) metric is developed to help design managers define the design maturity of an element based on its LOD level, performed design checks and reviews. In this regard, EDM completes the role of LOD, and fills the gap between modeling characteristics and design status.

Design Maturity Index (DMI) is another metric developed in this study to target the design maturity of the entire BIM model or part of it. Designers can, at any point in time, evaluate the design progress of the project by calculating its actual DMI. Accordingly, they can act upon the reasons hindering the project from reaching its desired maturity level, whether it is a modeling problem (not reaching desired LOD), or a process problem (missing design checks).

Moreover, the developed DFL method helps visualize the planning and control of the design process using the DMI metric. Each Information Package is planned to reach 100\% DMI at a certain duration, reflected by a planned DFL. Accordingly, design managers can visually plan the design process by specifying the Information Packages that need to be designed at each phase, their EDM requirements, their sequence based on information dependencies, their start/ end dates, and their durations.

Once the plan is set, design managers can follow up with design progress by calculating weekly DMIs and comparing them to the plan. Accordingly, deviations from the planned progress can be visually detected using real time data. Moreover, planners can balance the production of design information by balancing the production rates of involved design teams in order to avoid waste generation.

Nonetheless, the framework is able to capture the Transformation, Flow, and Value (TFV) aspects of design that are suggested by Koskela (2000). Transformation is reflected by elements converging to their required EDMs, thus transforming client needs to actual elements having defined design maturity levels. The Flow is highlighted as a flow of model elements; planned and controlled by the the DFL method, and the value aspect is targeted by the CI variable embedded in the EDM.

\section{REFERENCES}

Abou-Ibrahim, H. and Hamzeh, F., 2016. Enabling Lean Design Management: An LODBased Framework. Lean Construction Journal, pp. 12-24.

Al Hattab, M. and Hamzeh, F., 2016. Using social network theory and simulation to compare traditional versus BIM-lean practice for design error management. Automation in Construction , pp. 59-69.

Austin, S., Baldwin, A., Baizhan , L. and Waskett, P., 1999. Analytical Design Planning Technique: a model of the detailed building design process. Design Studies, Volume 20, pp. 279-296.

Autodesk, n.d. Autodesk.com. [Online] Available at: http://help.autodesk.com/view/RVT/2014/ENU/?guid=GUID-2480CA33C0B9-46FD-9BDD-FDE75B513727

Ballard, G., 2000. The last plannerTM system of production control. Birmingham: Ph.D. dissertation. The University of Birmingham.

BIMForum, 2015. Level of Development Specification, s.l.: s.n.

Cross, N., 1984. Developments in Design Methodology. Chichister: Wiley.

Eastman, C., Lee, J., Jeong, Y. and Lee, J., 2009. Automatic Rule-Based Checking of Building Designs. Elsevier, Automation in Construction, Volume 18, pp. 1011-1033.

Ford, D. and Sterman, J., 2003. The Liar's club: concealing rework in concurrent development. Concurrent Engineering: Research and Applications , 11(3), pp. 211-219. 
Gray, C. and Hughes, W., 2001. Building Design Management. s.l.:Oxford : Butterworth Heinemann.

Hammond, J. Choo, H.J., Austin, S., Tommelein, I.D., and Ballard, G., 2000. Integrating Design Planning, Scheduling, and Control With DePlan. 8th Annual Conference of the International Group for Lean Construction Brigthon, UK, s.n.

Hamzeh, F., Ballard, G. and Tommelein, I., 2009. Is the Last Planner System Applicable to Design? A Case Study. Taipei, Taiwan, Proc. 17th Ann. Conf. Int'l. Group for Lean Constr., IGLC 17, pp. 165-176.

Koskela, L. (2000). An exploration towards a production theory and its application to construction. Ph.D. Dissertation, Helsinki University of Technology.

Koskela , L., Huovila , P. and Leinonen , J., 2002. Design Management in Building Construction: from Theory to Practice. Journal of Construction Research, 3(1).

Krovi , R., Chandra , A. and Rajagopalan , B., 2003. Information flow parameters for managing organizational processes. Commun ACM, 46(2), pp. 77-82.

Mazlum, S. K. and Pekericli, M. K., 2016. Lean Design Management - an Evaluation of Waste Items for Architectural Design Process. METU JFK, 33(1), pp. 1-20.

Peirce, C. S., 1931. Collected papers of Charles Sanders Peirce / edited by Charles Hartshorne and Paul Weiss. s.l.:Cambridge : Harvard University Press.

The American Institute of Architects, 2013. Guide, Instructions and Commentary to the 2013 AIA Digital Practice Documents. s.l.:s.n.

Tribelsky, E. and Sacks, R., 2010. Measuring information flow in the detailed design of construction projects. Res Eng Design, Volume 21, p. 189-206.

Tribelsky, E. and Sacks, R., 2011. An Empirical Study of Information Flows in Multidisciplinary Civil Engineering Design Teams using Lean Measures. Architectural Engineering and Design Management, pp. 85-101.

Vaishnavi , V. K. and Kuechler, W., 2007. Design Science Research Methods and Patterns. s.l.:Auerbach Publications. 